\title{
WHEN DO THE UPPER KURATOWSKI TOPOLOGY (HOMEOMORPHICALLY, SCOTT TOPOLOGY) AND THE CO-COMPACT TOPOLOGY COINCIDE?
}

\author{
SZYMON DOLECKI, GABRIELE H. GRECO, AND ALOJZY LECHICKI
}

\begin{abstract}
A topology is called consonant if the corresponding upper Kuratowski topology on closed sets coincides with the co-compact topology, equivalently if each Scott open set is compactly generated. It is proved that Čechcomplete topologies are consonant and that consonance is not preserved by passage to $G_{\delta}$-sets, quotient maps and finite products. However, in the class of the regular spaces, the product of a consonant topology and of a locally compact topology is consonant. The latter fact enables us to characterize the topologies generated by some $\Gamma$-convergences.
\end{abstract}

\section{INTRODUCTION}

Let $\tau$ be topology on a set $X$. A filter $\mathscr{F}$ on $\mathbf{F}(\tau)$ (the family of all $\tau$-closed subsets of $X$ ) upper Kuratowski convergences to $A_{0} \in \mathbf{F}(\tau)$, if

$$
\bigcap_{F \in \mathscr{F}} \operatorname{cl}_{\tau}\left(\bigcup_{A \in F} A\right) \subset A_{0}
$$

The limit $\lim _{\tau^{*}} \mathscr{F}$ is the set of all the elements $\mathbf{F}(\tau)$ to which $\mathscr{F}$ converges in the above sense. Accordingly, we have defined a convergence $\tau^{*}$ on $\mathbf{F}(\tau)$. Given a convergence $\xi$ on a set $Y$, a subset $\mathscr{B}$ of $Y$ is $\xi$-closed if for every filter $\mathscr{F}$ on $\mathscr{B}(\mathscr{B} \in \mathscr{F}), \lim _{\xi} \mathscr{F} \subset \mathscr{B}$. The family of all $\xi$-closed sets generates a topology (induced by $\xi$ on $Y$ ) which we denote $T \xi$. In particular, $T \tau^{*}$ is the finest topology on $\mathbf{F}(\tau)$ for which $A_{0} \in \lim _{\tau^{*}} \mathscr{F}$ implies that every $\tau^{*}$-open set containing $A_{0}$ belongs to $\mathscr{F}$. We call it the upper Kuratowski topology (with respect to $\tau$ ). We identify each topology with the corresponding convergence: a filter converges to $y$ if it contains every (open) neighborhood of $y$. A convergence is said to be topological if it is equal to the topology it induces.

The co-compact topology $\tau^{\circ}$ on $\mathbf{F}(\tau)$ has the family

$$
\{A \in \mathbf{F}(\tau): A \cap K=\varnothing\},
$$

Received by the editors December 6, 1992 and, in revised form, December 24, 1993.

1991 Mathematics Subject Classification. Primary 54B20; Secondary 54A20, 06 B35.

Key words and phrases. Kuratowski convergence, co-compact topology, continuous convergence, Gamma convergence, compact families.

The first author's work has been partly supported by the Consiglio Nazionale delle Ricerche and the Deutscher Akademischer Austauschdienst. 
where $K$ ranges over $\tau$-compact subsets of $X$, for a base of open sets. It is well known and easy to see that in general $\tau^{*}$ is finer than $\tau^{\circ}$. Since $\tau^{\circ}$ is a topology, $T \tau^{*}$ is finer than $\tau^{\circ}$.

We say that a topology $\tau$ is consonant, if the corresponding upper Kuratowski and co-compact topologies coincide $\left(T \tau^{*}=\tau^{\circ}\right)$; in the opposite case $\tau$ is called dissonant.

1.1. Theorem. Let $\tau$ be a Hausdorff topology. The following assertions are equivalent: (i) the convergence $\tau^{*}$ is topological; (ii) $\tau^{*}=\tau^{\circ}$; (iii) $\tau$ is locally compact.

It is well known and straightforward that (iii) implies (ii). Of course, (ii) entails (i). That (i) implies (iii) follows from the theory of continuous lattices.

In [18] D. Scott introduces the topology (later called the Scott topology) induced on a complete lattice by the convergence

$$
x \leq \bigvee_{F \in \mathscr{F}} \bigwedge F
$$

He defines a continuous lattice as a complete lattice for which the above convergence is topological. It follows that the complete lattice $\mathscr{O}_{\tau}$ of $\tau$-open sets is continuous if and only if for every $x$ and each $U \in \mathscr{O}_{\tau}(x)$, the set of $\tau$ open sets that contain $x$, there is $W \in \mathscr{O}_{\tau}(x)$ such that each open cover of $U$ admits a finite subfamily that covers $W$. K. H. Hofmann and J. D. Lawson [14] call such topologies $\tau$ core-compact ${ }^{1}$ and show explicitly the above relationship ([5,16] for Hausdorff topologies). For Hausdorff spaces this condition is tantamount to the local compactness of $\tau$.

In the lattice $\mathbf{F}(\tau)$ (considered with $\leq$ equal to $\supset$ ) (1.3) amounts to the upper Kuratowski convergence (1.1). Therefore $\mathbf{F}(\tau)$ is continuous if and only if the upper Kuratowski convergence is topological. Consequently, Theorem 1.1 is a consequence of the just quoted results $[18,14,5,16]$.

A proof of Theorem 1.1 may be also deduced from those of the theorems by G. Choquet [4] and E. Lowen-Colebunders [17] that characterize the topologicity of the Kuratowski convergence (called sometimes the Kuratowski-Painlevé convergence [23]). A filter $\mathscr{F}$ on $\mathbf{F}(\tau)$ Kuratowski converges to $A_{0} \in \mathbf{F}(\tau)$, if, besides (1.1), for every $x \in A_{0}$ and every neighbourhood $Q$ of $x$, there is $F \in \mathscr{F}$ such that $A \cap Q \neq \varnothing$ for each $A \in F$. The latter condition combined with that of convergence in the co-compact topology amounts to the Fell topology [23].

This paper is devoted to the study of consonance. Primarily it explores phenomena that are not embraced by Theorem 1.1 shedding light on the outskirts of the theorem of Choquet and Lowen-Colebunders. Although we do not adopt a lattice theoretic approach, our results may contribute to the knowledge of a class of lattices broader than that of continuous lattices.

It turns out that $\tau^{*}$-open sets may be characterized in terms of $\tau$-compact families of sets. Denote $\mathscr{O}_{\tau}(A)=\left\{O \in \mathscr{O}_{\tau}: A \subset O\right\}$ and $\mathscr{O}_{\tau}(\mathscr{A})=\bigcup_{A \in \mathscr{A}} \mathscr{O}_{\tau}(A)$.

A family $\mathscr{A}=\mathscr{O}_{\tau}(\mathscr{A})$ is said to be compact if for every $\mathscr{Q} \subset \mathscr{O}_{\tau}$,

$$
\bigcup \mathscr{Q} \in \mathscr{A} \Rightarrow \underset{\mathscr{P} \subset \mathscr{Q}}{\exists}(|\mathscr{P}|<\infty \text { and } \bigcup \mathscr{P} \in \mathscr{A}) \text {. }
$$

\footnotetext{
${ }^{1}$ Core-compact topologies have been called differently by different authors: hereditarily locally compactoid in [10], semi-locally bounded by J. R. Isbell [16], quasi locally compact by A. S. Ward [20], "condition $C$ " by B. J. Day and G. M. Kelly [5].
} 
Such families are considered by Day and Kelly [5], J. R. Isbell [16] and, stable under finite intersections, by P. Wilker [21]. It follows from [18] that a subset of $\mathscr{O}_{\tau}$ is Scott open if and only if it is a $\tau$-compact family. For a family $\mathscr{G}$ of subsets of $X$, we denote $\mathscr{G}_{c}=\{X \backslash G: G \in \mathscr{G}\}$. Consequently a family $\mathscr{G} \subset \mathbf{F}(\tau)$ is open in the upper Kuratowski topology if and only if $\mathscr{G}_{c}=\mathscr{O}_{\tau}\left(\mathscr{G}_{c}\right)$ and $\mathscr{G}_{c}$ is $\tau$-compact (Theorem 3.1).

A family $\mathscr{A}=\mathscr{O}_{\tau}(\mathscr{A})$ is said to be compactly generated [21] whenever

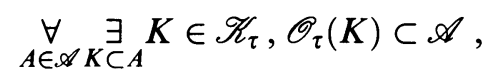

where $\mathscr{K}_{\tau}$ stands for the set of all $\tau$-compact subsets of $X$.

In view of (1.2), $\mathscr{G} \subset \mathbf{F}(\tau)$ is open in the co-compact topology if and only if $\mathscr{G}_{c}=\mathscr{O}_{\tau}\left(\mathscr{G}_{c}\right)$ and $\mathscr{G}_{c}$ is compactly generated.

Consequently, a topology $\tau$ is consonant if and only if each $\tau$-compact family $\mathscr{A}$ with $\mathscr{A}=\mathscr{O}_{\tau}(\mathscr{A})$ is compactly generated.

Many convergences on spaces of closed sets have been studied in the literature. The upper Kuratowski convergence and the co-compact topology have an especially important place among them.

The upper Kuratowski convergence formalizes the concept of closedness of multivalued functions that plays a fundamental role in stability questions of analysis and optimization; on the other hand, the upper Kuratowski convergence is the continuous convergence (with respect to the Sierpinski topology), that is the coarsest convergence that makes continuous the natural coupling between points and continuous mappings. In this particular case one identifies each subset $A$ of $X$ with a function $f$ valued in $Y=\{0,1\}$ to the effect that $A=\{x \in X: f(x)=0\}$. Now, consider the Sierpinski topology on $Y=\{0,1\}$ (its open sets are $\varnothing,\{0,1\}$ and $\{1\})$. Then continuous functions from $X$ to $Y$ correspond to closed subsets of $X$ and the resulting continuous convergence is equal to the upper Kuratowski convergence.

The co-compact topology is an instance of uniform convergence on compact sets whose role in functional analysis is well known to be fundamental. In fact, with the Sierpinski topology on $Y=\{0,1\}$ the compact-open topology on the set of all continuous functions from $X$ to $Y$ becomes the co-compact topology.

Therefore, the problem of consonance is a special case of the following general problem of the theory of continuous convergence and compact-open topologies: when is the compact-open topology the maximal "proper topology" on the set of the continuous functions? Indeed, the topologization of the continuous convergence is (almost by definition) the finest proper topology [2].

Our results provide a better understanding of $\Gamma$-convergence (another example of continuous convergences), a theory conceived by E. De Giorgi and $T$. Franzoni to cope with problems of calculus of variation [6], [7], of statistics (Wijsman [22]) and of other areas of mathematics.

We shall see that Čech-complete topologies (e.g., completely metrizable topologies, the Niemytzki plane) are consonant, but that consonance is not hereditary with respect to $G_{\delta}$ subspaces. However, consonance is hereditary with respect to closed and also hereditary with respect to open subspaces. Consonance turns out to be preserved neither by finite products nor by quotient maps. Challenging questions are open: whether metric spaces are consonant (for example, the set of rational numbers with the natural topology) or, more 
generally, whether regular $k$-topologies are consonant (for example, the Sorgenfrey line). An example of a $k$-topology which is dissonant but not regular is the radial topology of the plane (Section 5).

Studying consonance of topological spaces which are, a priori, either Hausdorff or completely regular does not make it easier to understand our results and, certainly, does not simplify their proofs. Therefore, in the sequel we do not assume any separation axioms for the underlying topologies. ${ }^{2}$ Consequently, throughout this paper, compact sets are not assumed to be Hausdorff. Therefore, all the compact sets (hence, not necessarily Hausdorff) take part in the definition of co-compact topology; verify the consonance of the topology on the real line generated by the sets of the form $]-\infty, r[$ to see the importance of that assumption. All other topological notions which are not explicitly redefined in this paper are the usual ones [12]. Thus regular spaces and locally compact spaces are assumed to be Hausdorff and Čech-complete spaces are assumed to be completely regular. However, we shall include occasional footnotes to clarify of the role of axioms of separation.

\section{NOTIONS OF COMPACTNESS}

As we have mentioned the topology $T \tau^{*}$ may be characterized in terms of $\tau$-compact families of sets. We shall first proceed to a review of some notions related to compactness (see $[5,16,19,21]$ as well as [10] and its bibliographical comments).

Let $\mathscr{A}$ and $\mathscr{B}$ be families of subsets of $X$. We write $\mathscr{A} \# \mathscr{B}$ whenever, for every $A \in \mathscr{A}$ and $B \in \mathscr{B}, A \cap B \neq \varnothing$. This amounts to $\mathscr{A} \subset \mathscr{B} \#$ (and to $\left.\mathscr{B} \subset \mathscr{A}^{\#}\right)$ where $\mathscr{B}^{\#}=\{H \subset X: H \cap B \neq \varnothing$ for each $B \in \mathscr{B}\}$ is the grill of $\mathscr{B}[4]$.

A family $\mathscr{D}$ is $\tau$-compactoid (resp. $\tau$-compact) if, for every filter $\mathscr{G}$ on $X$ such that $\mathscr{G} \# \mathscr{D}, \operatorname{adh}_{\tau} \mathscr{G} \neq \varnothing\left(\right.$ resp. $\left.\operatorname{adh}_{\tau} \mathscr{G} \in \mathscr{D}^{\#}\right)$, where $\operatorname{adh}_{\tau} \mathscr{G}=\bigcap_{G \in \mathscr{G}} \operatorname{cl}_{\tau} G$. These notions reduce to those of the relative compactness of a set $D$ (if $\tau$ is regular) and (resp.) to the compactness if we specialize $\mathscr{D}=\{D\} .^{3}$

Dually, $\mathscr{D}$ is a compactoid (resp. compact) family if and only if, for every family $\mathscr{Q}$ of open sets such that $\cup_{Q \in \mathscr{Q}} Q=X$ (resp. $\bigcup_{Q \in \mathscr{Q}} Q \supset D$ for some $D \in \mathscr{D})$, there exists $D_{0} \in \mathscr{D}$ and a finite subfamily of $\mathscr{Q}$, say $Q_{1}, \ldots, Q_{n}$, such that $\bigcup_{i=1}^{n} Q_{n} \supset D_{0}$.

For compactoid filters on regular topological spaces, we recall the following proposition [10].

2.1. Proposition. ${ }^{4}$ A filter $\mathscr{G}$ is compactoid if and only if adh $\mathscr{G}$ is compact and every open set $O \supset$ adh $\mathscr{G}$ belongs to $\mathscr{G}$.

It is very important to remember that compact families need not be filters; for example every family of compact sets is a compact family.

It is clear that a family $\mathscr{D}$ is compact if and only if $\mathscr{O}_{\tau}(\mathscr{D})$ is compact. For

\footnotetext{
${ }^{2}$ Of course, the resulting Kuratowski and co-compact topologies are not Hausdorff, except for $X=\varnothing$.

${ }^{3}$ Compact filters appear in [10], compact filters of open sets in [21] by P. Wilker and compact families of open sets in $[5,16]$ under different names; compactoid filters may be found under a different name in [19] by F. Topsøe as well as in [10] and in some earlier papers of the authors.

${ }^{4}$ The proposition holds also in the case where the required regularity does not include the Hausdorff property.
} 
compact filters on Hausdorff topological spaces, we have a useful characterization.

2.2. Proposition. A filter $\mathscr{G}$ is compact if and only if $\mathscr{G}$ is compactoid and $\operatorname{adh} \mathscr{G}=\bigcap_{G \in \mathscr{G}} G$. In particular, if a filter $\mathscr{G}$ is compact, then $\operatorname{adh} \mathscr{G}$ is compact and $\mathscr{O}(\operatorname{adh} \mathscr{G})=\mathscr{O}(\mathscr{G}) .^{5}$

\section{CRITERION FOR CONSONANCE}

Both the upper Kuratowski convergence and the co-compact topology may be described with the aid of reduced filters. If $\mathscr{F}$ is a filter on $2^{X}$ (or on some subset of $2^{X}$ ), then the reduced filter $|\mathscr{F}|$ is the filter on $X$ generated by the family $\left\{\bigcup_{A \in F} A: F \in \mathscr{F}\right\}$. Accordingly, $A \in \lim _{\tau^{*}} \mathscr{F}$ if and only if $A$ is $\tau$-closed and $\operatorname{adh}_{\tau}|\mathscr{F}| \subset A$. Moreover, $A \in \lim _{\tau^{\circ}} \mathscr{F}$ if and only if, for each compact set $K$ disjoint from (the $\tau$-closed set) $A$, there exists $H \in|\mathscr{F}|$ with $H \cap K=\varnothing$.

Although the following theorem is a consequence of a description of Scott open sets $[18,5]$, we provide here an alternative proof. Note that this theorem answers partially a question of $R$. Arens and J. Dugundji [2] concerning the characterization of open sets of continuous convergences in terms of the underlying topologies. For a given family $\mathscr{A}$ of subsets of $X$, let $\mathscr{A}_{c}=\left\{A^{c}: A \in \mathscr{A}\right\}$.

3.1. Theorem. A subset $\mathscr{A}$ of $\mathbf{F}(\tau)$ is open in the upper Kuratowski topology $T \tau^{*}$ if and only if $\mathscr{A}_{c}$ is a $\tau$-compact family such that $\mathscr{O}_{\tau}\left(\mathscr{A}_{c}\right)=\mathscr{A}_{c}$.

Proof. Let $\mathscr{A}$ be $\tau^{*}$-open. Then $\mathscr{A}_{c}$ is composed of $\tau$-open sets. Moreover if $A \in \mathscr{A}$ and $B$ is a $\tau$-closed set included in $A$, then $B \in \mathscr{A}$. Consequently, $\mathscr{O}_{\tau}\left(\mathscr{A}_{c}\right)=\mathscr{A}_{c}$. Suppose that $\mathscr{A}_{c}$ is not $\tau$-compact. Then, there exists a filter $\mathscr{G}$ on $X$ such that $\mathscr{G} \# \mathscr{A}_{c}$ and $\operatorname{adh}_{\tau} \mathscr{G} \notin\left(\mathscr{A}_{c}\right)^{\#}$. Since $\operatorname{adh}_{\tau} \mathscr{G}$ is $\tau$-closed, the latter means that $\operatorname{adh}_{\tau} \mathscr{G} \in \mathscr{A}$. Let $\mathscr{F}$ be the filter on $\mathbf{F}(\tau)$ generated by the family $\left\{B \in \mathbf{F}(\tau): B \subset \operatorname{cl}_{\tau} G\right\}_{G \in \mathscr{G}}$. Then $\operatorname{adh}_{\tau} \mathscr{G} \in \lim _{\tau^{*}} \mathscr{F}$ and since $\mathscr{A}$ is a $\tau^{*}$-open neighborhood of $\operatorname{adh}_{\tau} \mathscr{G}$, one has $\mathscr{A} \in \mathscr{F}$. In other words, there exists $G_{0} \in \mathscr{G}$ such that $\mathrm{cl}_{\tau} G_{0} \in \mathscr{A}$. On the other hand, $\mathscr{G} \# \mathscr{A}_{c}$ : for every $G \in \mathscr{G}$ and every $A \in \mathscr{A}, G \not \subset A$. Therefore $\operatorname{cl}_{\tau} G \notin \mathscr{A}$ for each $G \in \mathscr{G}, \mathrm{a}$ contradiction.

Conversely, suppose that $\mathscr{A}_{c}$ is a $\tau$-compact family fulfilling $\mathscr{O}_{\tau}\left(\mathscr{A}_{c}\right)=\mathscr{A}_{c}$. Hence if $D$ is a $\tau$-closed subset of an element of $\mathscr{A}$, then $D \in \mathscr{A}$. Let $\mathscr{F}$ be a filter on $\mathbf{F}(\tau) \backslash \mathscr{A}$. If $H$ is an element of the reduced filter $|\mathscr{F}|$, then there is $F \in \mathscr{F}$ such that $\bigcup_{A \in F} A \subset H$. Therefore $\mathrm{cl}_{\tau}(H) \notin \mathscr{A}$, since otherwise each closed subset of it would belong to $\mathscr{A}$ and thus $\mathscr{F}$ would be a subfamily of $\mathscr{A}$ contrary to the assumption. As a result, $\operatorname{cl}_{\tau}(H) \in\left(\mathscr{A}_{c}\right)^{\#}$, because otherwise there would be $A \in \mathscr{A}$ such that $A^{c} \cap \mathrm{cl}_{\tau}(H)=\varnothing$. Consequently, since the elements of $\mathscr{A}_{c}$ are $\tau$-open, $|\mathscr{F}| \# \mathscr{A}_{c}$. By compactness, $\operatorname{adh}_{\tau}|\mathscr{F}| \in\left(\mathscr{A}_{c}\right)^{\#}$; hence $\operatorname{adh}_{\tau}|\mathscr{F}| \notin \mathscr{A}$ proving that $\mathbf{F}(\tau) \backslash \mathscr{A}$ is $\tau^{*}$-closed. $\diamond$

Theorem 3.1 entails the following characterization of the closed sets for the upper Kuratowski topology.

3.2. Corollary. A subset $\mathscr{B}$ of $\mathbf{F}(\tau)$ is closed in the upper Kuratowski topology $T \tau^{*}$ if and only if the following properties hold:

\footnotetext{
${ }^{5}$ In the class of regular spaces (not necessarily Hausdorff) a filter $\mathscr{G}$ is compactoid if and only if the filter $\overline{\mathscr{G}}$ is compact, where $\overline{\mathscr{G}}$ denotes the filter generated by $\{\operatorname{cl} G: G \in \mathscr{G}$ \} [10].
} 
(3.1) if $B \in \mathscr{B}$ and $B^{\prime} \supset B$ is $\tau$-closed, then $B^{\prime} \in \mathscr{B}$;

(3.2) for every family $\left(Q_{i}\right)_{i}$ of $\tau$-open sets such that $\bigcup_{i} Q_{i} \in \mathscr{B}^{\#}$, there is a finite subfamily $Q_{i_{1}}, Q_{i_{2}}, \ldots, Q_{i_{n}}$ such that $\bigcup_{k=1}^{n} Q_{i_{k}} \in \mathscr{B}^{\#}$.

Proof. Let $\mathscr{B} \subset \mathbf{F}(\tau)$. Set $\mathscr{A}=\mathbf{F}(\tau) \backslash \mathscr{B}$. Observe that (3.1) holds if and only if $\mathscr{O}_{\tau}\left(\mathscr{A}_{c}\right)=\mathscr{A}_{c}$ or, equivalently, if and only if $\mathscr{A}_{c}=\mathscr{O}\left(\mathscr{B}^{\#}\right)$. Since (3.2) amounts to compactness of $\mathscr{B}^{\#}$, the statement follows from Theorem 3.1, because an open set $G$ is in $\mathscr{B}^{\#}$ if and only if $G^{c} \notin \mathscr{B}$; hence $G^{c} \in \mathscr{A}_{c}$. $\diamond$

We remark that $\mathscr{B} \subset \mathbf{F}(\tau)$ is closed in the co-compact topology if and only if (3.1) holds and for every $\tau$-open set $Q \in \mathscr{B}^{\#}$ there exists a $\tau$-compact set $K \subset Q$ such that $K \in \mathscr{B} \#$. Indeed, from the definition of the co-compact topology, $\mathscr{B}$ is closed if and only if $B \in \mathscr{B}$ provided that, for every $\tau$-compact set $K$ disjoint from $B$, there exists in $\mathscr{B}$ a $(\tau$-closed) set $A$ disjoint from $K$. By transposing the above statement, we infer that $\mathscr{O}(K) \subset \mathscr{B} \#$, but since $\mathscr{O}(K)$ is a $\tau$-compact filter and $\mathscr{B}$ consists of $\tau$-closed sets, $K \in \mathscr{B} \#$. Hence, in view of (3.2) one readily sees again that locally compact topologies are consonant. ${ }^{6}$

From Theorem 3.1 and Corollary 3.2 we infer that the sets of the form $\left(\mathscr{O}_{\tau}(\mathscr{D})\right)_{c}$ (resp. $\left.\mathscr{D}^{\#} \cap \mathbf{F}(\tau)\right)$, where $\mathscr{D}$ is a $\tau$-compact family, are all the open (resp. closed) sets in the upper Kuratowski topology.

It is straightforward that the sets of the form $\left(\mathscr{O}_{\tau}(\mathscr{K})\right)_{c}\left(\right.$ resp. $\left.\mathscr{K}^{\#} \cap \mathbf{F}(\tau)\right)$, where $\mathscr{K}$ is a family of $\tau$-compact sets, are all the open (resp. closed) sets in the co-compact topology. Consequently, we have the following criterion for consonance:

3.3. Theorem. A topology is consonant if and only if, for every compact family $\mathscr{D}$, there exists a family $\mathscr{K}$ of compact sets such that $\mathscr{O}(\mathscr{D})=\mathscr{O}(\mathscr{K})$. In other words, a topology is consonant if and only if for every nonempty compact family $\mathscr{D}$ with $\varnothing \notin \mathscr{D}$ and for every $Q \in \mathscr{O}(\mathscr{D})$, there exists a compact set $K \subset Q$ such that $\mathscr{O}(K) \subset \mathscr{O}(\mathscr{D})$.

We observe that if $\mathscr{D}$ is a compact filter, then by Proposition 2.2 the compact set $\operatorname{adh} \mathscr{D}$ is such that $\mathscr{O}(\mathscr{D})=\mathscr{O}(\operatorname{adh} \mathscr{D})$. Consequently, in the problem of deciding whether a given (Hausdorff) topology is consonant, we deal only with compact families that are not filters.

Notice that if the underlying topology is regular, then it is consonant if and only if every compact family $\mathscr{D}=\mathscr{O}(\mathscr{D})$ contains a compact filter. Indeed, if the topology is consonant and $\mathscr{D}$ is compact, then $\mathscr{D}=\bigcup_{K \in \mathscr{K}} \mathscr{O}(K)$ for some family $\mathscr{K}$ of nonvoid compact sets; the necessity follows, because each $\mathscr{O}(K)$ is a compact filter. Conversely, let $\mathscr{D}=\mathscr{O}(\mathscr{D})$ be a compact family and let $D_{0} \in \mathscr{D}$. Let $\mathscr{A}=\mathscr{O}\left(\left\{D \in \mathscr{D}: D \subset D_{0}\right\}\right)$. Then $\mathscr{A}$ is a compact family with $\mathscr{A}=\mathscr{O}(\mathscr{A})$ and by our assumption, there is a compact filter $\mathscr{G} \subset \mathscr{A}$. In view of Proposition 2.1 and by regularity, the compact filter $\mathscr{O}(\operatorname{adh} \mathscr{G})$ is included in $\mathscr{G}$; hence $\mathscr{O}(\operatorname{adh} \mathscr{G}) \subset \mathscr{A} \subset \mathscr{D}$ and $\operatorname{adh} \mathscr{G} \subset D_{0}$. This proves the consonance.

\section{SUFFICIENT CONDITIONS FOR CONSONANCE}

We have seen that locally compact topologies are consonant. We are going to extend this result to Čech-complete topologies. A topology is Čech-complete

\footnotetext{
${ }^{6}$ Actually it is sufficient that every point of the topological space admit a base of compact neighborhoods.
} 
if it is the restriction of a compact topology to a $G_{\delta}$-subset. We recall that a topology is Cech-complete if and only if it is completely regular and there exists a sequence $\left(\mathscr{B}_{n}\right)_{n}$ of open covers of the underlying space such that, whenever $\mathscr{F}$ is a family of closed sets with the finite intersection property such that for every $n, \mathscr{F} \cap \mathscr{B}_{n} \neq \varnothing$, then $\bigcap \mathscr{F} \neq \varnothing[12]^{7}$

\subsection{Theorem. ${ }^{8}$ Cech-complete topologies are consonant.}

Proof. Let $\left(\mathscr{B}_{n}\right)_{n}$ be a sequence of covers from the definition of Čechcompleteness. Let $\mathscr{D}$ be a nonempty compact family such that $\varnothing \notin \mathscr{D}$ and let $Q_{0} \in \mathscr{O}(\mathscr{D})$ be an open set. Since Čech-complete topologies are regular, the family of the nonempty open sets $A$ such that there is $B \in \mathscr{B}_{1}$ with cl $A \subset B \cap Q_{0}$ is an open cover of $Q_{0}$; thus by compactness of $\mathscr{D}$, there exists a nonempty open set $Q_{1} \in \mathscr{O}(\mathscr{D})$ and a finite subfamily of $\mathscr{B}_{1}$, say $B_{1}^{1}, B_{2}^{1}, \ldots, B_{k_{1}}^{1}$, such that $\operatorname{cl} Q_{1} \subset Q_{0}$ and $Q_{1} \subset \bigcup_{i=1}^{k_{1}} B_{i}^{1}$. Similarly, we can construct a sequence $Q_{2}, Q_{3}, \ldots Q_{n} \ldots$ of nonempty open sets such that, for every $n, Q_{n} \in \mathscr{O}(\mathscr{D}), \operatorname{cl} Q_{n+1} \subset Q_{n}$ and $Q_{n}$ is a a subset of a finite union of elements of $\mathscr{B}_{n}$. Now, let $\mathscr{G}$ be the filter base on $X$ composed by the sets $Q_{n}$. It is clear that adh $\mathscr{G} \subset Q_{0}=\bigcup_{n} Q_{n}$. Every ultrafilter $\mathscr{U}$ finer than $\mathscr{G}$ contains an element from every $\mathscr{B}_{n}$. Therefore, by Čech-completeness, $\mathscr{U}$ converges (this amounts to the compactoidness of $\mathscr{G}$ ). Using this fact and Proposition 2.1 (or by a direct argument) we have that the adherence adh $\mathscr{G}$ is compact and that for every open set $O$ that includes adh $\mathscr{G}$, there exists $n$ for which $Q_{n} \subset O$. By Theorem 3.1, this shows consonance. $\diamond$

Theorem 4.1 might suggest that consonance would be hereditary for $G_{\delta}$ subspaces. This however is not the case; in Example 7.2 we shall see a regular dissonant topology which is the restriction of a consonant topology to a $G_{\delta}$ subspace.

There are consonant regular topologies which are not Čech-complete, as we shall see later. A completely metrizable topology is Čech-complete, hence consonant. We do not know whether metrizable topologies (e.g. the usual topology of the set of rational numbers) are constant.

Čech-complete topologies are $k$-topologies. The only dissonant regular topologies we know are not $k$-topologies. The radial topology (see Section 5) is a dissonant $k$-topology which is not regular (if the dimension of the underlying vector space exceeds 1 ).

Let us observe that

4.2. Proposition. Consonance is hereditary with respect to open subspaces and with respect to closed subspaces.

Proof. Let $Y$ be a subset of a consonant topology on $X$. Let $\mathscr{D}$ be an arbitrary compact family on $Y$. Then $\mathscr{D}$ is a compact family on $X$ and thus there

\footnotetext{
${ }^{7}$ Let $\mathscr{H}$ be a family of closed subsets of a Čech-complete space, which has the finite intersection property, and let $\mathscr{G}$ denote the filter generated by $\mathscr{H}$. Clearly, adh $\mathscr{G}=\bigcap_{H \in \mathscr{X}} H$. $\mathscr{G}$ is compact (or, equivalently, compactoid; see Proposition 2.2) if and only if, for every $n, \mathscr{G}$ contains an element which is subset of a finite union of elements of $\mathscr{B}_{n}$. In particular, if, for every $n, \mathscr{H}$ contains sets included in some element of $\mathscr{B}_{n}$, then $\mathscr{G}$ is compact.

${ }^{8}$ Call quasi-Cech-complete a topology which admits a sequence $\left(\mathscr{B}_{n}\right)_{n}$ of open covers having the same property of Čech-complete topologies. Regular (not necessarily Hausdorff) quasi-Čechcomplete topologies are consonant. The proof of Theorem 4.1 works also in this case.
} 
exists a family $\mathscr{K}$ of compact subsets of $X$ for which $\mathscr{O}_{X}(\mathscr{D})=\mathscr{O}_{X}(\mathscr{K})$, where $\mathscr{O}_{X}$ refers to open sets of $X$. 1st case: $Y$ is open. Since a subset of $Y$ is compact (resp. open) in $Y$ if and only if it is compact (resp. open) in $X, \mathscr{O}_{Y}(\mathscr{D})=\mathscr{O}_{Y}\left(\mathscr{K} \cap 2^{X}\right)$. Hence $Y$ is consonant. 2 nd case: $Y$ is closed. Since $\mathscr{D}$ is a family of subsets of $Y, \mathscr{O}_{Y}(\mathscr{D})=\mathscr{O}_{X}(\mathscr{D}) \cap Y$, where for every family of sets $\mathscr{A}, \mathscr{A} \cap Y:=\{A \cap Y: A \in \mathscr{A}\}$. Moreover, it is clear that $\mathscr{O}_{X}(\mathscr{K}) \cap Y \subset \mathscr{O}_{Y}(\mathscr{K} \cap Y)$. On the other hand, since $Y$ is closed, one has that $\mathscr{O}_{X}(\mathscr{K}) \cap Y \supset \mathscr{O}_{Y}(\mathscr{K} \cap Y)$. Hence by $\mathscr{O}_{X}(\mathscr{D})=\mathscr{O}_{X}(\mathscr{K})$, one obtains $\mathscr{O}_{Y}(\mathscr{D})=\mathscr{O}_{Y}(\mathscr{K} \cap Y)$. As the elements of $\mathscr{K} \cap Y$ are compact, this shows the consonance of $Y$. $\diamond$

A point of a topological space is called a local compactness point (LC-point) if it admits a neighborhood base composed of compact sets.

Recall that a topology $\tau$ is said to be a $k_{1}$-topology if $x \in \mathrm{cl}_{\tau} A$ implies the existence of a compact set $K$ such that $x \in \operatorname{cl}_{\tau}(A \cap K)$ [3]. Recall as well that a topology is hemicompact if there exists a sequence of compact sets cofinal in the family of all compact sets ordered by inclusion [12]. One can easily prove that the co-compact topology $\tau^{\circ}$ is first-countable if and only if all the $\tau$-open sets are hemicompact. Without using the criterion of consonance (Theorem 3.3) we will prove the following

4.3. Theorem. Every Hausdorff $k_{1}$-topology in which all open sets are hemicompact is consonant.

Proof. Since hemicompactness of $\tau$-open sets implies that co-compact topology is first-countable, in order to prove the consonance it is enough to show that $\tau^{*}$-closed sets are sequentially closed in the co-compact topology which amounts to verifying that the convergence induced on sequences of $\tau$-closed sets by the co-compact topology is finer than the upper Kuratowski convergence. Let $A$ be a $\tau$-closed set and let $\left(A_{n}\right)_{n}$ be a sequence of $\tau$-closed sets which converges to $A$ in the co-compact topology. We need to prove that $\bigcap_{n} \mathrm{cl}_{\tau}\left(\bigcup_{m \geq n} A_{m}\right) \subset A$. If $\tau$ is Hausdorff, then the co-compact convergence of $\left(A_{n}\right)_{n}$ to $A$ means that, for every $\tau$-compact $K$,

$$
\bigcap_{n} \mathrm{cl}_{\tau}\left(K \cap \bigcup_{m \geq n} A_{m}\right) \subset A .
$$

Let $x_{0}$ be an arbitrary point belonging to $\bigcap_{n} \mathrm{cl}_{\tau}\left(\bigcup_{m \geq n} A_{m}\right)$. First case: for every $n, x_{0} \in \bigcup_{m \geq n} A_{m}$. Since $K=\left\{x_{0}\right\}$ is compact, from (4.1) it follows that $x_{0} \in A$. Second case: there is $n_{0}$ such that, for every $n \geq n_{0}, x_{0} \notin$ $A_{n}$. Since $x_{0} \in \operatorname{cl}_{\tau}\left(\bigcup_{m \geq n_{0}} A_{m}\right)$, there is a compact set $K$ such that $x_{0} \in$ $\mathrm{cl}_{\tau}\left(K \cap \bigcup_{m \geq n_{0}} A_{m}\right)$, because $\tau$ is a $k_{1}$-topology. As, for every $n \geq n_{0}, x_{0} \notin A_{n}$, we have that $x_{0} \notin \operatorname{cl}_{\tau}\left(K \cap A_{n}\right)$, because $\operatorname{cl}_{\tau}\left(K \cap A_{n}\right)$ is included in the closed set $A_{n}$. Since

$$
x_{0} \in \operatorname{cl}_{\tau}\left(K \cap \bigcup_{m \geq n_{0}} A_{m}\right)=\operatorname{cl}_{\tau}\left(K \cap \bigcup_{m \geq n} A_{m}\right) \cup \bigcup_{n_{0} \leq m \leq n} \operatorname{cl}_{\tau}\left(K \cap A_{m}\right),
$$

we obtain that, for every $n \geq n_{0}, x_{0} \in \mathrm{cl}_{\tau}\left(K \cap \bigcup_{m \geq n} A_{m}\right)$. Thus, by (4.1), $x_{0} \in A$. Therefore the sequence $\left(A_{n}\right)_{n} \tau^{*}$-converges to $A$. $\diamond$ 
4.4. Example. Let $\Omega$ be the following relation on $\mathbb{R}$ :

$$
\Omega r= \begin{cases}\mathbb{Z} & \text { if } r \in \mathbb{Z}, \\ \{r\} & \text { if } r \notin \mathbb{Z},\end{cases}
$$

where $\mathbb{Z}$ is the set of all integer numbers. Denote by $\xi$ the quotient of the natural topology $\nu$ on $\mathbb{R}$ by $\Omega$. The topology $\xi$ is known to be a Fréchet topology (see [12, Example 1.6.18]), hence $k_{1}$. The $\xi$-compact sets are the images by $\Omega$ of $\nu$-compact sets. Therefore $\xi$ is hereditary hemicompact with respect to open sets. Consequently it is consonant. We shall see in Example 7.2 that $\xi$ is not Čech-complete, so we could not use here Theorem 4.1.

\section{A DISSONANT $k$-TOPOLOGY}

A subset of a real vector space is closed with respect to the radial topology $\rho$ if its intersection with every straight line is closed in the usual topology of the real line. The radial topology is Hausdorff and induces the usual topology on every finite union of straight lines. A compact set (in particular, a convergent sequence) in the radial topology is included in a finite union of straight lines. ${ }^{9}$

5.1. Proposition. The radial topology is dissonant provided that the dimension of the underlying vector space is strictly greater than 1 .

Proof. Because of Proposition 4.2, it is enough to show that the plane equipped with the radial topology is dissonant. We are going to construct a sequence $\left(\mathscr{B}_{n}\right)_{n}$ of families of $\rho$-closed sets. A $\rho$-closed set $A$ belongs to $\mathscr{B}_{n}$ provided that the elements of $\left\{x:\|x\|=\frac{1}{n}\right\} \backslash A$ are distant one from another by at least $\frac{1}{n^{2}}$. Let $\mathscr{B}$ be the family of all $\rho$-closed sets that contain the origin.

For each $n, \mathscr{B}_{n}$ is $\rho^{*}$-closed. Indeed, we may consider the generating subfamily for $\mathscr{B}_{n}$ consisting of $\left\{x:\|x\|=\frac{1}{n}\right\}$ from which one has removed some points (with the distances not smaller than $\frac{1}{n^{2}}$ ). Let $\mathscr{F}$ be a filter on this subfamily. If $x_{1}, x_{2} \notin \operatorname{adh}_{\rho}|\mathscr{F}|$, then, in particular, there is $B$ belonging to the subfamily such that $x_{1}, x_{2} \notin B$ so that if $x_{1} \neq x_{2}$ and $\left\|x_{1}\right\|=\left\|x_{2}\right\|=\frac{1}{n}$, then $\left\|x_{1}-x_{2}\right\| \geq \frac{1}{n_{2}}$.

Next we observe that $\bigcap_{n} \bigcup_{m \geq n} \mathscr{B}_{n} \subset \mathscr{B}$. Indeed, if $B \notin \mathscr{B}$ and $B$ is $\rho$ closed, then $\{h:\|h\|=1\}=\bigcup_{n}\left\{h:\|h\|=1,\left[0, \frac{1}{n} h\right] \subset B^{c}\right\}$. Hence there is $n$ such that $\left\{h:\|h\|=1,\left[0, \frac{1}{n} h\right] \subset B^{c}\right\}$ infinite; therefore for each $m>n$, $B \notin \mathscr{B}_{m}$.

Finally, we claim that, for every $\rho$-compact set $K$, there exist $n_{0}$ and $B_{0} \in$ $\mathscr{B}_{n_{0}}$ such that $B_{0} \cap K=\varnothing$ (equivalently, such that $K \notin \mathscr{B}_{n_{0}}^{\#}$ ). Since $K$ is included in a finite union of straight lines, there are $n_{1}$ and a finite set $V \subset\left\{h:\|h\|=\frac{1}{n_{1}}\right\}$ such that $\left\{h:\|h\| \leq \frac{1}{n_{1}}\right\} \cap K \subset \bigcup_{h \in V}[0, h]$. Choose $n_{0} \geq n_{1}$ such that $\frac{d}{n_{0}} \geq \frac{1}{n_{0}^{2}}$, where $d=n_{1} \inf \left\{\left\|h-h^{\prime}\right\|: h \neq h^{\prime}\right.$ and $\left.h, h^{\prime} \in V\right\}$. Then $B_{0}=\left\{h:\|h\| \leq \frac{1}{n_{0}}\right\} \backslash K \in \mathscr{B}_{n_{0}}$.

The rest of the proof follows from the following general theorem.

\footnotetext{
${ }^{9}$ If a set $A$ is not a subset of any finite union of straight lines, then it contains an infinite subset $B$ that meets each straight line at most two points. Every subset of $B$ is $\rho$-closed. Hence $A$ is not compact.
} 
5.2. Theorem. Let $\tau$ be a topology. If there exist a sequence $\left(\mathscr{B}_{n}\right)_{n}$ of $\tau^{*}$-closed sets and $a \tau^{*}$-closed set $\mathscr{B} \neq \mathbf{F}(\tau)$ such that

$$
\bigcap_{n} \bigcup_{m \geq n} \mathscr{B}_{m} \subset \mathscr{B}
$$

and for each $\tau$-compact set $K \in \mathscr{B}^{\#}$, there exists $n$ for which $K \notin \mathscr{B}_{n}^{\#}$, then $\tau$ is dissonant.

Proof. We claim that $\mathscr{B} \cup \bigcup_{n} \mathscr{B}_{n}$ is $\tau^{*}$-closed. In view of Corollary 3.2, we need prove that $\left(\mathscr{B} \cup \cup_{n} \mathscr{B}_{n}\right)^{\#}$ is $\tau$-compact. Let $\mathscr{Q}$ be a family of $\tau$-open sets such that $\bigcup_{Q \in \mathscr{Q}} Q \in\left(\mathscr{B} \cup \cup_{n} \mathscr{B}_{n}\right)^{\#}=\mathscr{B}^{\#} \cap \bigcap_{n} \mathscr{B}_{n}^{\#}$. Since $\mathscr{B}$ is $\tau^{*}$ closed and $\bigcup_{Q \in \mathscr{Q}} Q \in \mathscr{B}^{\#}$, by Corollary 3.2 there is a finite subfamily $\mathscr{Q}^{\prime}$ of $\mathscr{Q}$ such that $\bigcup_{Q \in \mathscr{Q}^{\prime}} Q \in \mathscr{B}^{\#}$, which, by (5.1), is included in $\bigcup_{n} \bigcap_{m \geq n} \mathscr{B}_{m}^{\#}$; hence there is $n_{0}$ such that $\bigcup_{Q \in Q^{\prime}} Q \in \bigcap_{m \geq n_{0}} \mathscr{B}_{m}^{\#}$. On the other hand, since $\bigcup_{m=1}^{n_{0}} \mathscr{B}_{m}$ is $\tau^{*}$-closed and $\bigcup_{Q \in \mathscr{Q}} Q \in\left(\bigcup_{m=1}^{n_{0}} \mathscr{B}_{m}\right)^{\#}=\bigcap_{m=1}^{n_{0}} \mathscr{B}_{m}^{\#}$, by Corollary 3.2 there is a finite subfamily $\mathscr{Q}^{\prime \prime}$ of $\mathscr{Q}$ such that $\bigcup_{Q \in Q^{\prime \prime}} Q \in \bigcap_{m=1}^{n_{0}} \mathscr{B}_{m}^{\#}$. Hence $\bigcup_{Q \in \mathscr{Q}^{\prime} \cup \mathscr{Q}^{\prime \prime}} Q \in\left(\mathscr{B} \cup \bigcup_{n} \mathscr{B}_{n}\right)^{\#}$ showing that $\left(\mathscr{B} \cup \bigcup_{n} \mathscr{B}_{n}\right)^{\#}$ is $\tau$-compact.

Since $\mathscr{B} \neq \mathbf{F}(\tau)$ (i.e. $\varnothing \notin \mathscr{B})$, by virtue of $(5.1)$ we may assume that $\varnothing \notin \mathscr{B} \cup \bigcup_{n} \mathscr{B}_{n}$. The empty set belongs to the $\tau^{\circ}$-closure of $\mathscr{B} \cup \bigcup_{n} \mathscr{B}_{n}$. Indeed, it amounts to $K \notin\left(\mathscr{B} \cup \bigcup_{n} \mathscr{B}_{n}\right)^{\#}$, for every $\tau$-compact set $K$. But this is an immediate consequence of the assumptions. Therefore, the $\tau^{*}$-closed set $\mathscr{B} \cup \bigcup_{n} \mathscr{B}_{n}$ is not $\tau^{\circ}$-closed, because $\varnothing \notin \mathscr{B} \cup \bigcup_{n} \mathscr{B}_{n}$. $\diamond$

It follows from [8], [9] that if the dimension of the underlying real vector space is strictly greater than 1 , then the radial topology $\rho$ is not a Fréchet topology. Hence, from the description of $\rho$-compact sets we deduce that $\rho$ is not a $k_{1}$-topology under the dimension condition. It is more difficult to prove, always for dimension strictly greater than 1 , that $\rho$ is not regular (see [15]).

It is easy to see that the radial topology is a $k$-topology. Recall that Hausdorff $k$-topologies may be characterized as quotients of locally compact topologies [12]. Therefore we have

5.3. Proposition. Consonance is not preserved by quotient maps.

\section{COMPACT-REPELlENT POINTS}

A point $x_{0}$ of a topological space $(X, \tau)$ is called compact-repellent if it is not isolated and if it is not an accumulation point of any compact set; in other words, if for every $\tau$-compact set $K, x_{0} \notin \operatorname{cl}_{\tau}\left(K \backslash\left\{x_{0}\right\}\right)$.

Let $\left(F_{n}\right)_{n}$ be a sequence of $\tau$-closed subsets of $X$. We recall the usual definitions of upper and lower limits of $\left(F_{n}\right)_{n}$, denoted by $\mathbf{L s} F_{n}$ and $\mathbf{L i} F_{n}$, respectively. $\quad \mathbf{L s} F_{n}=\bigcap_{n} \operatorname{cl}_{\tau}\left(\bigcup_{m>n} F_{n}\right)$. On the other hand, $x \in \mathbf{L i} F_{n}$ if for every $\tau$-neighborhood $Q$ of $x$, there exists $n$ such that, for each $m \geq n, Q \cap$ $F_{m} \neq \varnothing$.

6.1. Theorem. A topology is dissonant if it admits a compact-repellent point $x_{0}$ and a sequence $\left(F_{n}\right)_{n}$ of closed sets such that $x_{0} \notin \bigcup_{n} F_{n}$ and

$$
\text { Ls } F_{n}=\mathbf{L i} F_{n}=\left\{x_{0}\right\} \text {. }
$$

Proof. Let $\mathscr{B}_{n}$ be the family of all the $\tau$-closed sets that include $F_{n}$ and $\mathscr{B}$ the family of all the $\tau$-closed sets containing $x_{0}$. These families are $\tau^{*}$-closed, 
because each $\tau^{*}$-limit of a filter $\mathscr{F}$ such that for some $F \in \mathscr{F}$, a $\tau$-closed set $C$ is included in $A$ for each $A \in F$, includes $C$.

We shall see that (6.1) implies (5.1). Let $B$ be a $\tau$-closed set with $B \in$ $\bigcap_{n} \cup_{m \geq n} \mathscr{B}_{n}$ : there exists a subsequence $\left(n_{k}\right)_{k}$ such that $B \in \mathscr{B}_{n_{k}}$ for each $k$. This amounts to $B \supset F_{n_{k}}$ for every $k$. It follows that $\operatorname{Ls} F_{n_{k}} \subset B$. Now $\left\{x_{0}\right\}=\mathbf{L i} F_{n} \subset \mathbf{L i} F_{n_{k}} \subset \mathbf{L s} F_{n_{k}}$, so that $x_{0} \in B$ and thus $B \in \mathscr{B}$.

Let $K$ be a $\tau$-compact set such that $K \in \mathscr{B}^{\#}$, that is, $x_{0} \in K$. As $x_{0}$ is compact-repellent, $K \backslash\left\{x_{0}\right\}$ is also, a $\tau$-compact set. Let $Q_{n}=$ $\left(\left\{x_{0}\right\} \cup \bigcup_{m \geq n} F_{m}\right)^{c}$. As Ls $F_{n} \subset\left\{x_{0}\right\}$, we have that $\bigcup_{n} Q_{n}=X \backslash\left\{x_{0}\right\}$ and $Q_{n}$ is open. Consequently, $\left(Q_{n}\right)_{n}$ is an open increasing cover of $K \backslash\left\{x_{0}\right\}$; thus there is $n_{0}$ such that $K \backslash\left\{x_{0}\right\} \subset Q_{n_{0}}$ and since $x_{0} \notin \bigcup_{n} F_{n}$, one has $K \cap \bigcup_{m \geq n_{0}} F_{m}=\varnothing$. This means, in particular, that $K \notin \mathscr{B}_{n_{0}}^{\#}$. $\diamond$

6.2. Example. One defines the Arens topology on $X=(] 0,1[\times] 0,1[) \cup\left\{x_{0}\right\}$, $x_{0}=(0,0)$, as follows: every $x \neq x_{0}$ is isolated; $Q$ is a neighborhood of $x_{0}$ if there exist $0<r_{0}<1$ and a function $\left.f:\right] 0, r_{0}[\rightarrow] 0,1$ [ such that

$$
\left\{(r, s): 0<s<f(r), 0<r<r_{0}\right\} \cup\left\{x_{0}\right\} \subset Q \text {. }
$$

This is a completely regular topology. Since compact sets are finite, one sees that $x_{0}$ is compact-repellent. On the other hand, $\left(F_{n}\right)_{n}$ defined by $\left.F_{n}=\left\{\frac{1}{n}\right\} \times\right] 0,1[$ fulfills the hypotheses of Theorem 6.1. Hence the Arens topology is dissonant. Thus [2, Theorem 6.5] amounts to this example. $\diamond$

6.3. Example (Naturalized Arens topology). The underlying set is the same as in the previous example 6.2. If the neighborhoods of $x \neq x_{0}$ are the neighborhoods of $x$ in the usual topology, $Q$ is a neighborhood of $x_{0}$ if there exist $0<r_{0}<1$ and a continuous function $\left.f:\right] 0, r_{0}[\rightarrow] 0,1[$ such that $\left\{(r, s): 0<s<f(r), 0<r<r_{0}\right\} \cup\left\{x_{0}\right\} \subset Q$. Compact sets in this topology are of the form $\left\{x_{0}\right\} \cup K$ or $K$, where $K$ is a compact subset of ]0,1[×]0,1[ in the usual topology. Consequently, $x_{0}$ is compact-repellent. On the other hand, $\left(F_{n}\right)_{n}$ defined by $F_{n}=\left\{\frac{1}{n}\right\} \times$ ]0, 1[ fulfills the hypotheses of Theorem 6.1. Hence the naturalized Arens topology is dissonant. Observe that the naturalized Arens topology is locally connected and that the compactrepellent point $x_{0}$ is a $G_{\delta}$-point because we may consider a sequence of neighborhoods $\left.Q_{n}:=\right] 0, \frac{1}{n}[\times] 0, \frac{1}{n}\left[\cup\left\{x_{0}\right\}\right.$ to the effect that $\left\{x_{0}\right\}=\bigcap_{n} Q_{n}$. Therefore to show its dissonance one may apply also the following corollary:

\subsection{Corollary. If a regular locally connected topology admits a compact-repellent} $G_{\delta}$-point, then it is dissonant. ${ }^{10}$

Proof. If $x_{0}$ is $G_{\delta}$ and the topology is regular, there exists a sequence $\left(W_{n}\right)_{n}$ of open sets such that $\operatorname{cl} W_{n+1} \subset W_{n}$ for each $n$ and $\left\{x_{0}\right\}=\bigcap_{n} W_{n}$. Let $F_{n}=$ $\operatorname{cl} W_{n} \backslash W_{n+1}$. Then Ls $F_{n} \subset \bigcap_{n} \operatorname{cl} W_{n}=\left\{x_{0}\right\}$ and $x_{0} \notin \bigcup_{n} F_{n}=\operatorname{cl} W_{1} \backslash\left\{x_{0}\right\}$. It suffices now to prove that $x_{0} \in \mathbf{L i} F_{n}$. Suppose that, on the contrary, $x_{0} \notin \mathbf{L i} F_{n}$ : there exist a connected neighborhood $Q$ of $x_{0}$ and a subsequence $\left(n_{k}\right)_{k}$ such that $Q \cap F_{n_{k}}=\varnothing$ for every $k$, that is, $Q \subset F_{n_{k}}^{c}=\left(\operatorname{cl} W_{n_{k}}\right)^{c} \cup W_{n_{k}+1}$. Since $Q$ is connected and meets $W_{n_{k}+1}, Q \subset W_{n_{k}+1}$ which leads to the contradiction: $Q=\left\{x_{0}\right\}$, but $x_{0}$ is not isolated.

\footnotetext{
${ }^{10}$ The corollary holds also in the case where the required regularity does not include the Hausdorff axiom.
} 
We shall see in the following example that there exist consonant topologies with compact-repellent points.

6.5. Example. Fix a point $x_{0}$ of an uncountable set $X$. One defines the topology co-countable at $x_{0}$ as follows: every $x \neq x_{0}$ is an isolated point and $Q$ is a neighborhood of $x_{0}$ whenever $x_{0} \in Q$ and the complement of $Q$ is countable. This is a regular topology for which every $G_{\delta}$-set is open and every compact set is finite. We shall see that it is consonant.

Let $\mathscr{D}=\mathscr{O}(\mathscr{D})$ be a compact family. Let $D \in \mathscr{D}$ be such that, for every compact set $K \subset D$, there is $A \in \mathscr{O}(K)$ such that $A \notin \mathscr{D}$. Consequently, $D$ must contain $x_{0}$. Let $V_{0}$ be an open neighborhood of $x_{0}$; then, $D \backslash V_{0}=\bigcup_{n} K_{n}$, for an increasing sequence $\left(K_{n}\right)_{n}$ of finite sets. We claim that there exists an increasing sequence of open sets $\left(Q_{n}\right)_{n}$ such that, for each $n, Q_{n} \in \mathscr{O}\left(K_{n} \cup\left\{x_{0}\right\}\right)$ and $Q_{n} \notin \mathscr{D}$. Indeed, as we have assumed that, for every compact set $K \subset D$, there is $A \in \mathscr{O}(K)$ such that $A \notin \mathscr{D}$, there is a sequence $\left(A_{n}\right)_{n}$ such that, for each $n, A_{n} \in \mathscr{O}\left(K_{n} \cup\left\{x_{0}\right\}\right)$ and $A_{n} \notin \mathscr{D}$. Clearly $Q_{n}=\bigcap_{m \geq n} A_{m}$ includes $K_{n} \cup\left\{x_{0}\right\}$ and is co-countable, hence open, because each $Q_{n}$ contains $x_{0}$. Finally, $Q_{n} \notin \mathscr{D}$, as otherwise $A_{n}$ would belong to $\mathscr{D}$. Since $\left(Q_{n}\right)_{n}$ is an increasing sequence of open sets out of $\mathscr{D}, V_{1}=\bigcup_{n} Q_{n} \notin \mathscr{D}$ by virtue of the compactness of $\mathscr{D}$. Then $V_{1}$ is an open neighborhood of $x_{0}$ and $D \subset$ $V_{1} \cup V_{0}$. Since $V_{1} \cap V_{0}$ is an open neighborhood of $x_{0}$, we can construct an open neighborhood $V_{2}$ of $x_{0}$ such that $V_{2} \notin \mathscr{D}$ and $D \subset V_{2} \cup\left(V_{1} \cap V_{0}\right)$. Similarly we can construct open sets $V_{3}, \ldots, V_{n}, \ldots$ such that, for every $n \geq 1, x_{0} \in V_{n}$, $V_{n} \notin \mathscr{D}$ and $D \subset V_{n+1} \cup\left(V_{n} \cap \cdots \cap V_{0}\right)$. Hence, by the compactness of $\mathscr{D}$, the open set $V=\bigcup_{n} \bigcap_{m \geq n} V_{m}$ does not belong to $\mathscr{D}$. On the other hand, $D \subset V$ and $D \in \mathscr{D}:$ a contradiction. Indeed if $x \notin V$, then there exists a strictly increasing sequence $\left(n_{k}\right)_{k}$ such that $x \notin V_{n_{k}}$ for every $k$; in particular, $x \notin V_{n_{2}} \cup V_{n_{1}} \supset V_{n_{2}} \cup\left(V_{n_{2}-1} \cap \cdots \cap V_{n_{1}} \cap \cdots \cap V_{0}\right) \supset D$. $\diamond$

\section{Products}

Clearly, if the product of two topological spaces $X$ and $Y$ is consonant, then they are consonant. Conversely, the product of two consonant topologies need not be consonant (see Example 7.2). However

7.1. Theorem. The product of a regular consonant topology with a locally compact topology is consonant.

Proof. ${ }^{11}$ Let $X$ be equipped with a regular consonant topology and $Y$ with a locally compact topology. Let $\mathscr{D}$ be a compact family on $X \times Y$ such that $\varnothing \notin \mathscr{D}$ and let $Q \in \mathscr{O}_{X \times Y}(\mathscr{D})$. Pick an open cover $\mathscr{B}$ of $Q$ such that the projection $p_{Y}(B)$ on $Y$ of every element $B$ of $\mathscr{B}$ is relatively compact and $\bigcup_{B \in \mathscr{B}} \operatorname{cl}_{X \times Y} B=Q$. As $\mathscr{D}$ is compact, there exists a finite subfamily, say $B_{1}, B_{2}, \ldots, B_{n}$, of $\mathscr{B}$ such that $W=\bigcup_{i=1}^{n} B_{i} \in \mathscr{O}_{X \times Y}(\mathscr{D})$. Of course, $p_{Y}(W)$ is relatively compact in $Y$ and $\operatorname{cl}_{X \times Y} W \subset Q$. Sure enough, $\mathscr{A}=$ $\{B \in \mathscr{O}(\mathscr{D}): B \subset W\}$ is a compact family on $W$ satisfying $\mathscr{O}_{W}(\mathscr{A})=\mathscr{A}$. The projection $p_{X}(\mathscr{A})$ is a compact family on $p_{X}(W)$. By Proposition 4.2, $p_{X}(W)$ is consonant. Consequently, since $\varnothing \notin p_{X}(\mathscr{A})$, there exists a nonempty

\footnotetext{
${ }^{11}$ This proof works also in the case of the product of a regular (not necessarily Hausdorff) consonant topology with a topology for which every point admits a base of closed compact neighborhoods.
} 
compact subset $K$ of $p_{X}(W)$ such that $\mathscr{O}_{p_{X}(W)}(K) \subset \mathscr{O}_{p_{X}(W)}\left(p_{X}(\mathscr{A})\right)$. For every $H \in \mathscr{O}_{p_{X}(W)}(K)$, there exists $A_{H} \in \mathscr{A}$ such that $H=p_{X}\left(A_{H}\right)$. The subfamily $\left\{\bigcup\left\{A_{H}: H \subset C, H \in \mathscr{O}_{p_{X}(W)}(K)\right\}\right\}_{C \in O_{p_{X}(W)}(K)}$ of $\mathscr{A}$ generates a filter $\mathscr{G}$ on $W$. Since $p_{X}(\mathscr{G})=\mathscr{O}_{p_{X}(W)}(K)$ and the elements of $p_{Y}(\mathscr{G})$ are subsets of the relatively compact set $p_{Y}(W)$, the filter bases $p_{X}(\mathscr{G})$ and $p_{Y}(\mathscr{G})$ are compactoid, so that $\mathscr{G}$ is compactoid on $X \times Y$, because its projections are compactoid [10, Theorem 5.1]. Hence, by Proposition 2.1, adh $\mathscr{G}$ is a compact subset of $X \times Y$ and $\mathscr{O}_{X \times Y}(\operatorname{adh} \mathscr{G}) \subset \mathscr{O}_{X \times Y}(\mathscr{G})$. In view of Theorem 3.3, the consonance is proved. $\diamond$

Now, we exhibit an example of the product of a Čech-complete topology with a consonant topology (both being Fréchet) which is not constant.

7.2. Example. Denote by $\tau$ the topology induced on $X=\mathbb{R} \backslash\left\{\frac{1}{n}: 0 \neq n \in \mathbb{N}\right\}$ by the usual topology $\nu$ of $\mathbb{R}$. Let $\xi$ be the topology of Example 4.4 on $Y$. We follow now Example 2.4.20 of R. Engelking [12] showing that $\tau \times \xi$ is not a $k$-topology. Let

$$
F_{n}=\bigcup_{k \geq n}\left\{x \in X:\left|\frac{1}{n}-x\right| \leq \frac{1}{k}\right\} \times\left\{n-\frac{1}{k}\right\} .
$$

Each $F_{n}$ is $\tau \times \xi$-closed and $\mathbf{L s} F_{n}=\mathbf{L i} F_{n}=\{(0, \mathbf{0})\}$, where $\mathbf{0}$ is the equivalence class of 0 (from Example 4.4). On the other hand, if $K$ is $\tau \times \xi$-compact, then its projection on $Y \backslash\{0\}$ is bounded, so that $\{(0,0)\}$ is compact-repellent. Therefore we may apply Theorem 6.1 to conclude that $\tau \times \xi$ is dissonant. $\diamond$

From Example 7.2 it follows, as claimed in Example 4.4, that the topology $\xi$ is not Čech-complete, due to the fact that the products of two (actually, countably many) Čech-complete topologies are Čech-complete. On the other hand, $\tau \times \xi$ is the restriction to a $G_{\delta}$-set of the consonant topology $\nu \times \xi$ on $\mathbb{R} \times Y$. Therefore we have

7.3. Proposition. Consonance is not hereditary with respect to $G_{\delta}$-subspaces.

\section{SOME $\Gamma$-CONVERGENCES}

Theorem 7.1 enables us to characterize the finest topology that is coarser than the $\Gamma$-convergence of lower semicontinuous extended real valued functions. This convergence plays a fundamental role for the stability of the sets of minima [6], [7]. Let $\tau$ be a topology on a set $X$ and denote by $C_{-}(\tau)$ the set of all functions $f: X \rightarrow \overline{\mathbb{R}}$ which are lower $\tau$-semicontinuous with respect the usual topology on $\overline{\mathbb{R}}$. Let $\mathscr{F}$ be a filter on $C_{-}(\tau)$. It $\Gamma(\tau)$-converges to $f_{0} \in C_{-}(\tau)$ if, for each $x_{0} \in X$,

$$
\sup _{Q \in \mathscr{N}_{\tau}\left(x_{0}\right)} \sup _{F \in \mathscr{F}} \inf _{f \in F} \inf _{x \in Q} f(x) \geq f_{0}\left(x_{0}\right)
$$

where $\mathscr{N}_{\tau}\left(x_{0}\right)$ stands for the family of all neighborhoods of $x_{0}$ in $\tau$. Consider now the lower uniform-on-compact-sets topology of lower semicontinuous functions: a filter $\mathscr{F}$ on $C_{-}(\tau)$ converges to $f_{0} \in C_{-}(\tau)$ for this topology if, for every $\tau$-compact set $K$,

$$
\sup _{F \in \mathscr{F}} \inf _{f \in F} \inf _{K} f \geq \inf _{K} f_{0}
$$


where $\inf _{K} f=\inf _{x \in K} f(x)$. We denote this topology on $C_{-}(\tau)$ by $\Gamma_{c}(\tau)$.

If $f: X \rightarrow \overline{\mathbb{R}}$, then the epigraph of $f$ is defined by

$$
\text { epi } f=\{(x, r) \in X \times \mathbb{R}: f(x) \leq r\} .
$$

Let $\operatorname{Epi}(\tau)$ be the set of all the epigraphs of lower $\tau$-semicontinuous functions. Denote by $\nu_{+}$the topology on $\mathbb{R}$ consisting of all open intervals of the form ] $-\infty, a\left[\right.$. One readily sees that $\mathbf{F}\left(\tau \times \nu_{+}\right)=\mathbf{E p i}(\tau) \subset \mathbf{F}(\tau \times \nu)$ and that $\left(\tau \times \nu_{+}\right)^{*}$ is the restriction of $(\tau \times \nu)^{*}$ to $\operatorname{Epi}(\tau)$.

It is well known that $\Gamma(\tau)$-convergence of lower semicontinuous functions amounts to the upper Kuratowski convergence $(\tau \times \nu)^{*}$ of their epigraphs. Therefore

8.1. Proposition. The mapping epi from $\left(C_{-}(\tau), \Gamma(\tau)\right)$ onto $\left(\mathbf{E p i}(\tau),\left(\tau \times \nu_{+}\right)^{*}\right)$ is a convergence homeomorphism. ${ }^{12}$

Moreover, if $\tau$ is a regular topology, then we have

8.2. Proposition. The mapping epi from $\left(C_{-}(\tau), \Gamma_{c}(\tau)\right)$ onto $(\mathbf{E p i}(\tau)$, $\left.\left(\tau \times \nu_{+}\right)^{\circ}\right)$ is a homeomorphism.

Proof. The sets

$$
\left\{f \in C_{-}(\tau): \inf _{K} f>r\right\},
$$

where $K$ is $\tau$-compact and $r$ is a real number, constitute a subbase of open sets for $\Gamma_{c}(\tau)$. The images of the sets (8.3) by the mapping epi are

$$
\left.\left.\left\{A \in \mathbf{F}\left(\tau \times \nu_{+}\right): A \cap(K \times]-\infty, r\right]\right)=\varnothing\right\} .
$$

They are $\left(\tau \times \nu_{+}\right)^{\circ}$-open, because $\left.]-\infty, r\right]$ is $\nu_{+}$-compact. Hence epi is continuous. To complete the proof it is enough to show that the sets of the form (8.4) constitute a subbase of open sets for $\left(\tau \times \nu_{+}\right)^{\circ}$. To this end, consider

$$
\text { \{epi } \left.f: f \in C_{-}(\tau) \text {, epi } f \cap H=\varnothing\right\},
$$

where $H$ is a $\left(\tau \times \nu_{+}\right)$-compact set. This is an element of a base of $(\tau \times$ $\left.\nu_{+}\right)^{\circ}$. Fix a function $f_{0}$ such that epi $f_{0}$ belongs to (8.5). For every $(x, s) \notin$ epi $f_{0}$, there exist a neighborhood $Q$ of $x$ and $r>s$ such that epi $f_{0} \cap(Q \times$ $\mathrm{]}-\infty, r])=\varnothing$. Since the projection $p_{X}(H)$ of $H$ is a compact regular subspace of $(X, \tau)$, there is a $P \in \mathscr{N}_{\tau}(x), P \subset Q$ such that $P \cap p_{X}(H)$ is compact. The sets $\left.\operatorname{int}_{\tau}\left(P \cap p_{X}(H)\right) \times\right]-\infty, r[$ form an open cover of the compact set $H$, because epi $f_{0}$ is $\left(\tau \times \nu_{+}\right)$-closed. Consequently, there are a finite number of $\tau$-compact sets $K_{1}, K_{2}, \ldots, K_{m}$ and of real numbers $r_{1}, r_{2}, \ldots, r_{m}$ such that $\left.\left.H \subset \bigcup_{i=n}^{m}\left(K_{i} \times\right]-\infty, r_{i}\right]\right)$ and epi $\left.\left.f_{0} \cap\left(K_{i} \times\right]-\infty, r_{i}\right]\right)=\varnothing$ for each $i=1,2, \ldots, m$. Therefore

$$
\begin{aligned}
\text { epi } f_{0} & \left.\left.\in \bigcap_{i=1}^{m}\left\{\text { epi } f: f \in C_{-}(\tau) \text {, epi } f_{0} \cap\left(K_{i} \times\right]-\infty, r_{i}\right]\right)\right\} \\
& \subset\left\{\text { epi } f: f \in C_{-}(\tau), \text { epi } f \cap H=\varnothing\right\} .
\end{aligned}
$$

Since the sets of the form $]-\infty, r]$ are compact in $\nu_{+}$, the proof is complete. $\diamond$

Denote by $T \Gamma(\tau)$ the finest topology coarser than the convergence $\Gamma(\tau)$ (i.e. $T \Gamma(\tau)$ is a topology on $C_{-}(\tau)$ whose closed sets are the closed sets for $\left.\Gamma(\tau)\right)$.

\footnotetext{
${ }^{12} \mathrm{An}$ injective mapping $h$ from a convergence space onto another convergence space is a convergence homeomorphism if $x \in \lim \mathscr{F}$ if and only if $h(x) \in \lim h(\mathscr{F})$.
} 
8.3. Theorem. Let $\tau$ be a regular topology. ${ }^{13}$ Then the topologies $T \Gamma(\tau)$ and $\Gamma_{c}(\tau)$ coincide if and only if $\tau$ is constant.

Proof. In view of Propositions 8.1 and 8.2 it is enough to show that $\tau \times \nu_{+}$is consonant if and only if $\tau$ is consonant. If $\tau \times \nu_{+}$is consonant, it is obvious that $\tau$ is consonant. Conversely, let $\tau$ be consonant and let $\mathscr{B} \subset \mathbf{F}\left(\tau \times \nu_{+}\right)$ be an arbitrary $\left(\tau \times \nu_{+}\right)^{*}$-closed set. We claim that $\mathscr{B}$ is $\left(\tau \times \nu_{+}\right)^{\circ}$-closed. Set $\mathscr{W}=\left\{F \in \mathbf{F}(\tau \times \nu): \exists_{B \in \mathscr{B}} B \subset F\right\}$. Observe that $\mathscr{B}^{\#}=\mathscr{W}^{\#}$ and that epi $f \cap(Q \times] r, s[) \neq \varnothing$ amounts to epi $f \cap(Q \times]-\infty, s[) \neq \varnothing$, for every $Q \subset$ $X, f \in C_{-}(\tau)$ and for every pair $r, s$ of real numbers. Consequently, since $\mathscr{B}^{\#}$ is a $\left(\tau \times \nu_{+}\right)$-compact family, $\mathscr{W}^{\#}$ is also a $(\tau \times \nu)$-compact family. Therefore, by Corollary 3.2, $\mathscr{W}$ is $(\tau \times \nu)^{*}$-closed. As $(\tau \times \nu)$ is consonant (see Theorem $7.1)$, the family $\mathscr{W}$ is $(\tau \times \nu)^{\circ}$-closed. Finally, to show that $\mathscr{B}$ is $\left(\tau \times \nu_{+}\right)^{\circ}$ closed, take an arbitrary $f_{0} \in C_{-}(\tau)$ such that epi $f_{0} \notin \mathscr{B}$. By the definition of $\mathscr{W}$, we have that epi $f_{0} \notin \mathscr{W}$. As $\mathscr{W}$ is $(\tau \times \nu)^{\circ}$-closed, there is a $(\tau \times \nu)$ compact (hence $\left(\tau \times \nu_{+}\right)$-compact) set $H$ such that epi $f_{0} \cap H=\varnothing$ and $\mathscr{W} \cap$ $\{F \in \mathbf{F}(\tau \times \nu): F \cap H=\varnothing\}=\varnothing$. Therefore $\mathscr{B}$ is $\left(\tau \times \nu_{+}\right)^{\circ}$-closed. $\diamond$

Now, we shall see that the convergence $\Gamma(\tau)$ and the topology $\Gamma_{c}(\tau)$ are instances of continuous convergence and compact-open topology, respectively.

Let $\bar{\nu}_{-}$denote the topology on $\overline{\mathbb{R}}$ consisting of all intervals of the form ] $a,-\infty]$ and let $C\left(\tau, \bar{\nu}_{-}\right)$be the set of all functions $f: X \rightarrow \overline{\mathbb{R}}$ which are continuous when $\overline{\mathbb{R}}$ is equipped with $\bar{\nu}_{-}$. Observe that $C\left(\tau, \bar{\nu}_{-}\right)=C_{-}(\tau)$ and that the convergence $\Gamma(\tau)$ is the coarsest convergence $\zeta$ on $C_{-}(\tau)$ for which the canonical coupling ${ }^{14}\langle\rangle:,(X, \tau) \times\left(C_{-}(\tau), \zeta\right) \rightarrow\left(\overline{\mathbb{R}}, \bar{\nu}_{-}\right)$is jointly continuous. Therefore $\Gamma(\tau)$ is the continuous convergence on $C\left(\tau, \bar{\nu}_{-}\right)$. On the other hand, one readily sees that the lower uniform-on-compact-sets topology $\Gamma_{c}(\tau)$ is the compact-open topology on $C\left(\tau, \bar{\nu}_{-}\right)$.

\section{REFERENCES}

1. R. Arens, A topology for spaces of transformations, Ann. of Math. (2) 47 (1946), 480-495.

2. R. Arens and J. Dugundji, Topologies for function spaces, Pacific J. Math. 1 (1951), 5-31.

3. E. Čech, Topological spaces, Academia, Prague, 1959.

4. G. Choquet, Convergences, Ann. Univ. Grenoble 23 (1947-48), 57-112.

5. B. J. Day and G. M. Kelly, On topological quotient maps, Proc. Cambridge Philos. Soc. 67 (1970), 553-559.

6. E. De Giorgi and T. Franzoni, Su un tipo di convergenza variazionale, Atti Accad. Naz. Lincei 58 (1975), 842-850.

7. E. De Giorgi, Generalized limits in calculus of variations, Topics in Functional Analysis, 1980-81, Quaderni della Scuola Norm. Sup. di Pisa, 1982.

8. S. Dolecki and G. H. Greco, Cyrtologies of convergences. II: Sequential convergences, Math. Nachr. 127 (1986), 317-334.

9. __ Topologically maximal pretopologies, Studia Math. 77 (1983), 265-281.

10. S. Dolecki, G. H. Greco and A. Lechicki, Compactoid and compact filters, Pacific J. Math. 117 (1985), 69-98.

11. _ Sur la topologie de la convergence supérieure de Kuratowski, C. R. Acad. Sci. Paris 312 (1991), 923-926.

${ }^{13}$ This theorem and Proposition 8.2 hold also in the case where the required regularity does not include the Hausdorff axiom.

${ }^{14}$ The canonical coupling is defined by $\langle x, f\rangle=f(x)$. 
12. R. Engelking, General topology, PWN, Warszawa, 1977.

13. J. Fell, A Hausdorff topology for closed subsets of a locally compact non-Hausdorff space, Proc. Amer. Math. Soc. 13 (1962), 472-476.

14. K. H. Hofmann and J. D. Lawson, The spectral theory of distributive continuous lattices, Trans. Amer. Math. Soc. 246 (1978), 285-310.

15. S. P. Franklin, Solution of problem 5468 (S. W. Williams), Amer. Math. Monthly 74 (1967), 207.

16. J. R. Isbell, Meet-continuous lattices, Sympos. Math. 16 (1975), 41-54.

17. E. Lowens-Colebunders, On the convergence of closed and compact sets, Pacific J. Math. 108 (1983), 133-139.

18. D. Scott, Continuous lattices, Lecture Notes in Math., vol. 274, Springer, New York, 1972, pp. 97-136.

19. F. Topsoe, Compactness in spaces of measures, Studia Math. 36 (1970).

20. A. S. Ward, Problem in "Topology and its applications" (Proc. Herceg., Nov. 1968), Belgrade, 1969, p. 352.

21. P. Wilker, Adjoint product and HOM functors in general topology, Pacific J. Math. 34 (1970), 269-283.

22. R. Wijsman, Convergence of sequences of convex sets, cones and functions. II, Trans. Amer. Math. Soc. 123 (1966), 32-45.

23. G. Beer, Topologies on closed and closed convex sets, Kluwer Academic, 1993.

Département de Mathematiques, Universite de Bourgogne, B.P. 138, 21004 Dijon Cedex, France and Institut für Statistik und Mathematische Wirtschaftstheorie, Universität Karlsruhe, Kollegium am Ehrenhof, 7500 Karlsruhe, Germany

Dipartimento di Matematica, Università di Trento, 38050 Povo, Italy

GeSELLSCHAFT FÜR INFORMATIONSTECHNIK, LUITPOLDSTRASSE 83, D-91052 ERLANGEN, GERMANY 